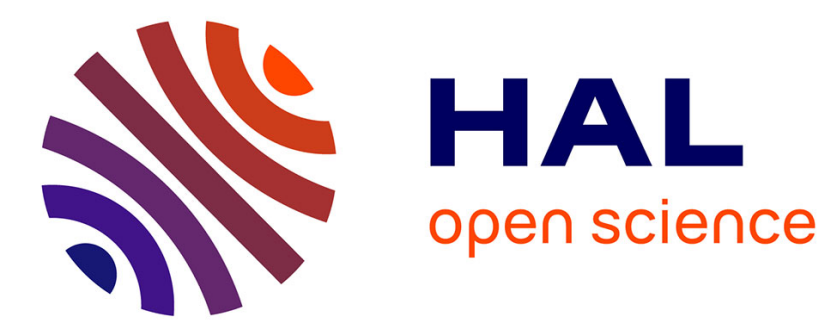

\title{
MOMENTS OF A THUE-MORSE GENERATING FUNCTION
}

\author{
Christian Mauduit, Hugh L. Montgomery, Joel Rivat
}

\section{To cite this version:}

Christian Mauduit, Hugh L. Montgomery, Joel Rivat. MOMENTS OF A THUE-MORSE GENERATING FUNCTION. 2019. hal-02278731

\section{HAL Id: hal-02278731 \\ https://hal.science/hal-02278731}

Preprint submitted on 4 Sep 2019

HAL is a multi-disciplinary open access archive for the deposit and dissemination of scientific research documents, whether they are published or not. The documents may come from teaching and research institutions in France or abroad, or from public or private research centers.
L'archive ouverte pluridisciplinaire HAL, est destinée au dépôt et à la diffusion de documents scientifiques de niveau recherche, publiés ou non, émanant des établissements d'enseignement et de recherche français ou étrangers, des laboratoires publics ou privés. 


\title{
MOMENTS OF A THUE-MORSE GENERATING FUNCTION
}

\author{
CHRISTIAN MAUDUIT, HUGH L. MONTGOMERY, AND JOËL RIVAT
}

ABSTRACT. We study the moments of even order of the generating function $\prod_{0 \leq r<n}\left(1-e\left(2^{r} x\right)\right)$ of the Thue-Morse sequence and we present several conjectures related to these moments.

\section{INTRODUCTION}

For any nonnegative integer $m$ we denote by $s(m)$ the number of distinct powers of 2 in the binary representation of $m$. Then the Thue-Morse sequence (or Prouhet-Thue-Morse sequence) is the sequence $\left((-1)^{s(m)}\right)_{m \in \mathbb{N}}$. This sequence occurs in many questions related to combinatorics, algebra, number theory, harmonic analysis, geometry, dynamical systems, ergodic theory, etc. (see for example [1] or [14]). Mahler introduced it for the first time in the context of harmonic analysis in [13] in order to illustrate the results obtained by Wiener [19]. He showed in particular that for any $k \in \mathbb{N}$ the sequence $\left(\frac{1}{N} \sum_{m<N}(-1)^{s(m)}(-1)^{s(m+k)}\right)_{N \in \mathbb{N}}$ converges, and that the limit is nonzero for infinitely many integers $k$. This can be interpreted as the fact that the correlation measure of the symbolic dynamical system associated to the Thue-Morse sequence is the Riesz product $\prod_{r>0}\left(1-\cos 2^{r} t\right)($ see $[12])$.

It follows from the definition of $s$ that for $|z|<1$ we have

$$
\prod_{r=0}^{\infty}\left(1-z^{2^{r}}\right)=\sum_{m=0}^{\infty}(-1)^{s(m)} z^{m}
$$

For any $n \in \mathbb{N}$ we consider the function $T_{n}$ defined for $x \in \mathbb{R}$ by

$$
T_{n}(x)=\prod_{0 \leq r<n}\left(1-e\left(2^{r} x\right)\right)=\sum_{0 \leq m<2^{n}}(-1)^{s(m)} e(m x),
$$

where $e(\theta)=e^{2 \pi i \theta}$.

The study of the values of $\left\|T_{n}\right\|_{p}, 1 \leq p \leq+\infty$ plays an important role in many problems. In particular, sharp estimates for $\left\|T_{n}\right\|_{1}$ and $\left\|T_{n}\right\|_{\infty}$ allowed Mauduit and Rivat to prove a Prime Number Theorem for the sum-of-digits function in [15] (see [16] and [5] for the study of the sumof-digits function along polynomial sequences) and the study of the ratios $\left\|T_{n+1}\right\|_{p} /\left\|T_{n}\right\|_{p}$ (for $p$ an even integer) allowed Kurths, Pikowsky, and Zaks to compute the generalized dimension for the Fourier spectrum of the Thue-Morse sequence in [21] (see also [20]).

Here we consider the moments

$$
M_{k}(n)=\int_{0}^{1}\left|T_{n}(x)\right|^{2 k} d x
$$

Date: February 9, 2016.

2010 Mathematics Subject Classification. Primary 11B85, $11 \mathrm{~B} 37$.

Key words and phrases. Thue-Morse sequence, generating function, moments, linear recurrence.

First author supported in part by Ciencia sem Fronteiras project PVE 407308/2013-0, second author supported in part by NSF grant DMS-063529, first and third authors supported in part by Agence Nationale de la Recherche project ANR-10-BLAN 0103 MUNUM. 
for positive integers $k$, which turn out to have some interesting and unexpected properties. By Parseval's identity it is clear that $M_{k}(n)$ is an integer, and that $M_{1}(n)=2^{n}$. We show that for any fixed $k$, the sequence of moments $M_{k}(n)$ satisfies a linear recurrence of order $k$.

Theorem 1. For positive integers $k$ let $A_{k}=\left[a_{i j}\right]$ be the $k \times k$ matrix with integral entries

$$
a_{i j}=(-1)^{i} 2^{2 k-2 i-1}\left(\begin{array}{c}
j+k-i \\
i
\end{array}\right) \frac{j+k}{j+k-i} \quad(0 \leq i, j<k),
$$

and let

$$
p_{k}(z)=\operatorname{det}\left(z I-A_{k}\right)=z^{k}-c_{k-1, k} z^{k-1}-\cdots-c_{0, k} \in \mathbb{Z}[z]
$$

be the characteristic polynomial of $A_{k}$. Then

$$
M_{k}(n)=c_{k-1, k} M_{k}(n-1)+\cdots+c_{0, k} M_{k}(n-k)
$$

for all integers $n \geq k$.

The fact that the $a_{i j}$ are integers is not immediately clear from (1.3), but their integrality will be established in the course of the proof of Theorem 1.

Theorem 2. For all positive integers $k$, there exists a $C_{k}>0$ such that for $n \rightarrow+\infty$,

$$
M_{k}(n)=C_{k} \rho\left(A_{k}\right)^{n}(1+o(1))
$$

where $\rho\left(A_{k}\right)$ denotes the spectral radius of $A_{k}$.

It is well known that $\left\|T_{n}\right\|_{\infty} \leq 2 \times 3^{(n-1) / 2}$ (see for example [8, Lemme II], [17, Lemma p. 72-73] or $[6$, Formule $(2.10)])$ so that

$$
M_{k}(n) \leq\left(\frac{4}{3}\right)^{k} \times 3^{k n}
$$

and it follows from (1.6) that

$$
\rho\left(A_{k}\right) \leq 3^{k}
$$

The next theorem gives a better upper bound for $\rho\left(A_{k}\right)$.

Theorem 3. We have

$$
\rho\left(A_{k}\right) \leq \frac{1}{2}\left(3^{k}+4^{2 k / 3}\right)=\frac{3^{k}}{2}(1+o(1))
$$

The method used in the proof of Theorem 2 yields better upper bounds for $\rho\left(A_{k}\right)$ for small values of $k$. For example it leads to $\rho\left(A_{2}\right) \leq 4 \sqrt{2}$ and $\rho\left(A_{3}\right) \leq 16$, while in fact numerical computation gives $\rho\left(A_{2}\right)=5.1231$ and $\rho\left(A_{4}\right)=14.2191$ and we conjecture that $\rho\left(A_{k}\right) \sim \frac{1}{2} 3^{k}$ (see section 7 ).

It would be nice to have more explicit formulas for the $c_{j, k}$ defined by (1.4). In this direction, we determine the trace and determinant of $A_{k}$, which yields $c_{k-1, k}$ and $c_{0, k}$.

Theorem 4. With the matrix $A_{k}$ defined as in (1.3),

$$
\operatorname{tr} A_{k}=3^{k-1}+(-1)^{k-1}
$$

and

$$
\operatorname{det} A_{k}=\varepsilon_{k} 2^{k^{2}}
$$

where $\varepsilon_{k}=1$ if $k \equiv 0$ or $1(\bmod 4)$, and $\varepsilon_{k}=-1$ if $k \equiv 2$ or $3(\bmod 4)$. 
From (1.7) it follows that if $t_{k}=\operatorname{tr} A_{k}$, then

$$
t_{k}=2 t_{k-1}+3 t_{k-2} .
$$

Since $c_{0, k} \neq 0$, the linear recurrence (1.5) is genuinely of order $k$ (not less). However, it could still be the case that $M_{k}$ satisfies a linear recurrence of lower order - in which case the polynomial $p_{k}$ would be reducible. Numerical experimentation suggests that the $p_{k}$ are all irreducible, but this is far from proven. Indeed, numerical experimentation suggests that the eigenvalues of the $A_{k}$, and the coefficients of $p_{k}$ have many striking properties. Our conjectures on this issue are collected in $\S 7$.

The authors are happy to thank Dick Askey for helpful comments, particularly relating to the identities in $\S 2$.

\section{A COMBINATORIAL IDENTITY}

In the course of proving Theorem 1, we encounter the following combinatorial sum, which can be written in closed form.

Lemma 1. For integers $i$ and $n$ with $0 \leq i<n$,

$$
s_{0}(n, i):=\sum_{i \leq m \leq n / 2}\left(\begin{array}{c}
n \\
2 m
\end{array}\right)\left(\begin{array}{c}
m \\
i
\end{array}\right)=2^{n-2 i-1}\left(\begin{array}{c}
n-i \\
i
\end{array}\right) \frac{n}{n-i} .
$$

This formula is asserted as item (3.120) in Gould [9, p. 36], but with no indication as to where a proof might be found. A complicated proof can be pieced together by combining several exercises from various chapters of Riordan [18]. A machine proof might be constructed using an implementation of Zeilberger's algorithm, but we have not achieved that. In fact, the formula is ancient, as it is a special case of a formula known to Chu in the thirteenth century (see Askey [2, Chapter 7]).

Proof. Let $r=m-i$. Then

$$
s_{0}(n, i)=\left(\begin{array}{c}
n \\
2 i
\end{array}\right) \sum_{r=0}^{n / 2-i} \frac{\left(\begin{array}{c}
n \\
2 r+2 i
\end{array}\right)\left(\begin{array}{c}
r+i \\
i
\end{array}\right)}{\left(\begin{array}{c}
n \\
2 i
\end{array}\right)}=\left(\begin{array}{c}
n \\
2 i
\end{array}\right) \sum_{r=0}^{n / 2-i} a_{r},
$$

say. By simple algebra we see that

$$
\frac{a_{r+1}}{a_{r}}=\frac{(r+i-n / 2)(r+i-(n-1) / 2)}{(r+1)(r+i+1 / 2)} .
$$

Since $a_{0}=1$, it follows that

$$
\begin{aligned}
s_{0}(n, i) & =\left(\begin{array}{c}
n \\
2 i
\end{array}\right) \sum_{r=0}^{n / 2-i} \frac{(i-n / 2)_{r}(i-(n-1) / 2)_{r}}{(i+1 / 2)_{r} r !} \\
& =\left(\begin{array}{c}
n \\
2 i
\end{array}\right){ }_{2} F_{1}\left(\begin{array}{c}
i-n / 2, i-(n-1) / 2 \\
i+1 / 2
\end{array}\right) .
\end{aligned}
$$

Here $(x)_{r}=x(x+1)(x+2) \cdots(x+r-1)$ is the Pochhammer symbol.

The Chu-Vandermonde identity asserts that if $s$ is a nonnegative integer, then

$$
\sum_{r=0}^{s} \frac{(-s)_{r}(a)_{r}}{(c)_{r} r !}=\frac{(c-a)_{s}}{(c)_{s}}
$$

for arbitrary $a$ and $c$. In Andrews-Askey-Roy [3, Corollary 2.2.3] this arises as a special case of a hypergeometric identity due to Gauss [7]. 
Suppose that $n$ is even, say $n=2 t$. In (2.3) we take $s=t-i, a=i-t+1 / 2$, and $c=i+1 / 2$ to see that

$$
s_{0}(n, i)=\left(\begin{array}{c}
n \\
2 i
\end{array}\right) \frac{(t)_{t-i}}{(i+1 / 2)_{t-i}} .
$$

Now $(a+1 / 2)_{m}=(2 a+1)_{2 m} /\left(2^{2 m}(a+1)_{m}\right)$, so that

$$
s_{0}(n, i)=2^{2 t-2 i}\left(\begin{array}{c}
n \\
2 i
\end{array}\right) \frac{(t)_{t-i}(i+1)_{t-i}}{(2 i+1)_{2 t-2 i}} .
$$

As $(t)_{t-i}=(n-i-1) ! /(t-1) !,(i+1)_{t-i}=t ! / i !$, and $(2 i+1)_{2 t-2 i}=n ! /(2 i) !$, we get

$$
s_{0}(n, i)=2^{n-2 i} \frac{(n-i-1) ! t}{i !(n-2 i) !}=2^{n-2 i-1}\left(\begin{array}{c}
n-i \\
i
\end{array}\right) \frac{n}{n-i},
$$

as was to be shown.

Suppose that $n$ is odd, say $n=2 t+1$. In (2.3) we take $s=t-i, a=i-t-1 / 2$, and $c=i+1 / 2$ to see that

$$
\begin{aligned}
s_{0}(n, i) & =\left(\begin{array}{c}
n \\
2 i
\end{array}\right) \frac{(t+1)_{t-i}}{(i+1 / 2)_{t-i}}=2^{2 t-2 i}\left(\begin{array}{c}
n \\
2 i
\end{array}\right) \frac{(t+1)_{t-i}(i+1)_{t-i}}{(2 i+1)_{2 t-2 i}} \\
& =2^{n-2 i-1} \frac{n(2 t-i) !}{i !(n-2 i) !}=2^{n-2 i-1}\left(\begin{array}{c}
n-i \\
i
\end{array}\right) \frac{n}{n-i},
\end{aligned}
$$

which completes the proof.

The sum $s_{0}(n, i)$ has a companion, namely

$$
s_{1}(n, i):=\sum_{i \leq m \leq(n-1) / 2}\left(\begin{array}{c}
n \\
2 m+1
\end{array}\right)\left(\begin{array}{c}
m \\
i
\end{array}\right)=2^{n-2 i-1}\left(\begin{array}{c}
n-i-1 \\
i
\end{array}\right) .
$$

This evaluation in closed form is also an easy consequence of the Chu-Vandermonde identity (2.3). By using familiar properties of binomial coefficients it is easy to show that

$$
\begin{aligned}
& s_{0}(n+1, i)=s_{0}(n, i)+s_{1}(n, i)+s_{1}(n, i-1), \\
& s_{1}(n+1, i)=s_{1}(n, i)+s_{0}(n, i) .
\end{aligned}
$$

These identities make it possible to prove (2.1) and (2.4) simultaneously by a double induction. This is a little tedious, since various bases of induction need to be checked. In addition, this ignores the fact that both (2.1) and (2.4) are simple consequences of an ancient formula.

\section{Proof of Theorem 1}

Following Fouvry \& Mauduit [6], for $f \in L^{2}(\mathbb{T})$ we define the operators

$$
\begin{aligned}
P_{k} f(x) & =\frac{1}{2}(2 \sin \pi x / 2)^{2 k} f(x / 2)+\frac{1}{2}(2 \cos \pi x / 2)^{2 k} f((x+1) / 2), \\
Q_{k} f(x) & =(2 \sin \pi x)^{2 k} f(2 x) .
\end{aligned}
$$

Thus

$$
M_{k}(n)=\int_{0}^{1} Q_{k}^{n} 1 d x
$$


For $f, g \in L^{2}(\mathbb{T})$ we note that

$$
\begin{aligned}
\left\langle Q_{k} f, g\right\rangle & =\int_{0}^{1}(2 \sin \pi x)^{2 k} f(2 x) \overline{g(x)} d x \\
& =\frac{1}{2} \int_{0}^{2}(2 \sin \pi u / 2)^{2 k} f(u) \overline{g(u / 2)} d u \\
& =\frac{1}{2} \int_{0}^{1} f(u)\left((2 \sin \pi u / 2)^{2 k} \overline{g(u / 2)}+(2 \cos \pi u / 2)^{2 k} \overline{g((u+1) / 2)}\right) d u \\
& =\left\langle f, P_{k} g\right\rangle .
\end{aligned}
$$

Thus $P_{k}$ is the adjoint of $Q_{k}, P_{k}=Q_{k}^{*}$. In particular,

$$
M_{k}(n)=\left\langle Q_{k}^{n} 1,1\right\rangle=\left\langle 1, P_{k}^{n} 1\right\rangle=\int_{0}^{1} P_{k}^{n} 1 d x .
$$

Let $E_{k}$ denote the vector space of even trigonometric polynomials with period 1 and degree $<k$. Of course $\cos 2 \pi j x$ for $0 \leq j<k$ is a basis for $E_{k}$, but we note that

$$
\sin ^{2 j} \pi x=(-1)^{j} 2^{-2 j} \sum_{n=-j}^{j}(-1)^{n}\left(\begin{array}{c}
2 j \\
j-n
\end{array}\right) e(n x)
$$

is an even trigonometric polynomial with period 1 and degree $j$, so $1, \sin ^{2} \pi x, \sin ^{4} \pi x, \ldots$, $\sin ^{2(k-1)} \pi x$ is also a basis for $E_{k}$. Suppose that $0 \leq j<k$. Then

$$
P_{k} \sin ^{2 j} \pi x=2^{2 k-1} \sin ^{2(j+k)} \frac{\pi x}{2}+2^{2 k-1} \cos ^{2(j+k)} \frac{\pi x}{2} .
$$

By the half angle formulæ this is

$$
=2^{2 k-1}\left(\frac{1-\cos \pi x}{2}\right)^{j+k}+2^{2 k-1}\left(\frac{1+\cos \pi x}{2}\right)^{j+k} .
$$

By the binomial theorem this is

$$
=2^{k-j} \sum_{0 \leq m \leq(j+k) / 2}\left(\begin{array}{c}
j+k \\
2 m
\end{array}\right) \cos ^{2 m} \pi x,
$$

which is an even trigonometric polynomial with period 1 and degree $[(j+k) / 2]<k$. Thus $P_{k}$ maps $E_{k}$ to itself. Let $\widetilde{P_{k}}$ denote the restriction of $P_{k}$ to $E_{k}$. Continuing from (3.6), we find that

$$
\begin{aligned}
\widetilde{P_{k}} \sin ^{2 j} \pi x & =2^{k-j} \sum_{0 \leq m \leq(j+k) / 2}\left(\begin{array}{c}
j+k \\
2 m
\end{array}\right)\left(1-\sin ^{2} \pi x\right)^{m} \\
& =2^{k-j} \sum_{0 \leq m \leq(j+k) / 2}\left(\begin{array}{c}
j+k \\
2 m
\end{array}\right) \sum_{i=0}^{m}(-1)^{i}\left(\begin{array}{c}
m \\
i
\end{array}\right) \sin ^{2 i} \pi x \\
& =2^{k-j} \sum_{0 \leq i \leq(j+k) / 2}(-1)^{i} \sin ^{2 i} \pi x \sum_{i \leq m \leq(j+k) / 2}\left(\begin{array}{c}
j+k \\
2 m
\end{array}\right)\left(\begin{array}{c}
m \\
i
\end{array}\right) .
\end{aligned}
$$

Here it is clear that the coefficient of $\sin ^{2 i} \pi x$ is an integer. From Lemma 1 with $n=j+k$, we see that

$$
\widetilde{P_{k}} \sin ^{2 j} \pi x=\sum_{i=0}^{k-1} a_{i j} \sin ^{2 i} \pi x
$$


with the $a_{i j}$ defined in (1.3). Let $p_{k}$ be the characteristic polynomial of the matrix $A_{k}=\left[a_{i j}\right]$, as defined in (1.4). By the Cayley-Hamilton theorem we know that $p_{k}\left(A_{k}\right)=0$. Thus

$$
A_{k}^{n}=c_{k-1, k} A_{k}^{n-1}+c_{k-2, k} A_{k}^{n-2}+\cdots+c_{0, k} A_{k}^{n-k}
$$

for $n \geq k$, and hence

$$
\widetilde{P}^{n}=c_{k-1, k}{\widetilde{P_{k}}}^{n-1}+c_{k-2, k} A \widetilde{P}_{k}^{n-2}+\cdots+c_{0, k}{\widetilde{P_{k}}}^{n-k}
$$

Thus

$$
\begin{aligned}
M_{k}(n) & =\int_{0}^{1}{\widetilde{P_{k}}}^{n} 1 d x \\
& =\int_{0}^{1} c_{k-1, k}{\widetilde{P_{k}}}^{n-1} 1+\cdots+c_{0, k}{\widetilde{P_{k}}}^{n-k} 1 d x \\
& =c_{k-1, k} M_{k}(n-1)+c_{k-2, k} M_{k}(n-2)+\cdots+c_{0, k} M_{k}(n-k),
\end{aligned}
$$

which completes the proof.

\section{Proof of Theorem 2}

The operator $P_{k}$ that we introduced in (3.1) is a special case of positive quasi-compact transfer operators that have been studied by many authors in ergodic theory (see in particular $[4,10,11]$ ).

When the transfer function is a trigonometric polynomial (in our case $(2 \sin \pi x)^{2 k}$ ) the quasicompactness of $P_{k}$ is trivial. Indeed, as we saw in $\S 3$ the operator $P_{k}$ acts on the $k$ dimensional vector space $E_{k}$ and $A_{k}$ is the matrix of $P_{k}$ in the basis $\left(1, \sin ^{2} \pi x, \sin ^{4} \pi x, \ldots, \sin ^{2(k-1)} \pi x\right)$.

Proposition 1. The spectral radius of $P_{k}$ is equal to

$$
\rho\left(A_{k}\right)=\lim _{n \rightarrow+\infty}\left\|P_{k}^{n} 1\right\|_{\infty}^{1 / n}
$$

and is the only eigenvalue of $P_{k}$ with modulus $\rho\left(A_{k}\right)$. The eigenfunction $\psi_{k}$ associated to $\rho\left(A_{k}\right)$ is strictly positive on $[0,1]$. We have the following spectral decomposition of $P_{k}$ :

$$
E_{k}=\operatorname{ker}\left(P_{k}-\rho\left(A_{k}\right) \mathrm{Id}\right) \oplus F_{k},
$$

where $F_{k}$ is a subspace of $E_{k}$ stabilized by $P_{k}$ and such that the spectral radius of the restriction of $P_{k}$ to $F_{k}$ is strictly less than $\rho\left(A_{k}\right)$. Moreover for any $x \in[0,1]$ we have

$$
\rho\left(A_{k}\right)=\lim _{n \rightarrow+\infty} \frac{P_{k}^{n+1} 1(x)}{P_{k}^{n} 1(x)} .
$$

Proof. Proposition 1 follows from the study made by Hervé in [11] in the context of wavelet theory (see in particular Théorème 3.1 and Théorème 4.2 from [11]). In order to apply the results from [11]) it is enough to check that the function $(2 \sin \pi x)^{2 k}$ does not admit any invariant periodic cycle (i.e. there exists no positive integer $Q$ such that

$$
\left.\forall q \in\{1, \ldots, Q\}, \forall \ell \in\left\{0, \ldots, 2^{q}-1\right\}, \quad \sin \pi\left(\frac{\ell}{2^{q}-1}+\frac{1}{2}\right)=0\right),
$$

The fact that $\psi_{k}$ is stricly positive is a consequence of the study by Conze and Raugi in [4] on the invariant compact sets associated to the transformations $x \mapsto \frac{x}{2}$ and $x \mapsto \frac{x+1}{2}$ of the interval $[0,1]$ and the description by Hervé in [10] of the zeros of the eigenvalues of this class of operators. Moreover the fact that $\rho\left(A_{k}\right)$ is the only eigenvalue of $P_{k}$ with modulus $\rho\left(A_{k}\right)$ follows from the proof of Théorème 4.2 in [11]. 
Writing the function 1 according to (4.2), it follows that

$$
1=\alpha_{k} \psi_{k}+f_{k},
$$

with $\alpha_{k} \in \mathbb{R}\left(\alpha_{k} \neq 0\right.$ because of (4.1)) and $f_{k} \in F_{k}$.

This implies that

$$
\begin{aligned}
M_{k}(n) & =\int_{0}^{1} P_{k}^{n} 1 d x \\
& =\alpha_{k} \rho\left(A_{k}\right)^{n} \int_{0}^{1} \psi_{k}(x) d x\left(1+O\left(\theta_{k}^{-n}\right)\right),
\end{aligned}
$$

where $\theta_{k}>1$ is the ratio of $\rho\left(A_{k}\right)$ to the spectral radius of the restriction of $P_{k}$ to $F_{k}$.

\section{Proof of Theorem 3}

Applying the method of [17, Lemma pages 72-73] or [6, page 583] we have for all integer $n \geq 2$

$$
T_{n}(x)=\prod_{0 \leq r<n}\left|2 \sin \left(\pi 2^{r} x\right)\right| \leq 2 \prod_{0 \leq r<n-1} u\left(2^{r} x\right),
$$

where

$$
u(x)=2|\sin (\pi x)|^{2 / 3}|\sin (2 \pi x)|^{1 / 3} .
$$

It follows that

$$
M_{k}(n) \leq 4^{k} I_{k}(n-1)
$$

where for $n \geq 0$,

$$
I_{k}(n)=\int_{0}^{1} \prod_{0 \leq r<n} u\left(2^{r} x\right)^{2 k} d x
$$

(with the convention that an empty product is equal to 1 , so that $I_{k}(0)=1$ ). We have for $n \geq 1$

$$
\begin{aligned}
I_{k}(n) & =\int_{0}^{1 / 2} u(x)^{2 k} \prod_{1 \leq r<n} u\left(2^{r} x\right)^{2 k} d x+\int_{1 / 2}^{1} u(x)^{2 k} \prod_{1 \leq r<n} u\left(2^{r} x\right)^{2 k} d x \\
& =\int_{0}^{1}\left(\frac{1}{2} u(x / 2)^{2 k}+\frac{1}{2} u((x+1) / 2)^{2 k}\right) \prod_{0 \leq r<n-1} u\left(2^{r} x\right)^{2 k} d x \\
& \leq w_{k} I_{k}(n-1),
\end{aligned}
$$

where

$$
w_{k}=\max _{x \in \mathbb{R}} \frac{1}{2}\left(u(x / 2)^{2 k}+u((x+1) / 2)^{2 k}\right) .
$$

By induction we get

$$
I_{k}(n) \leq w_{k}^{n} I_{k}(0)=w_{k}^{n} .
$$

For all $x \in \mathbb{R}$ we have $u(x / 2)^{3}+u((x+1) / 2)^{3}=8|\sin \pi x| \leq 8$, so that $\min (u(x / 2), u((x+1) / 2)) \leq$ $4^{1 / 3}$. Furthermore for all $x \in \mathbb{R}$ we have $u(x) \leq u(1 / 3)=\sqrt{3}$, hence

$$
w_{k} \leq \frac{1}{2}\left(3^{k}+4^{2 k / 3}\right) \text {. }
$$

It follows that $M_{k}(n) \leq 4^{k} I_{k}(n-1) \leq 4^{k} w_{k}^{n-1}$ and from (1.6) we deduce that

$$
\rho\left(A_{k}\right) \leq w_{k},
$$

which completes the proof of Theorem 3 . 


\section{Proof of Theorem 4}

From the definition (1.3) of the $a_{i j}$ it is clear that

$$
\begin{aligned}
\operatorname{tr} A_{k} & =\sum_{i=0}^{k-1} a_{i i}=\frac{1}{k} \sum_{i=0}^{k-1}(-1)^{i} 2^{2 k-2 i-1}\left(\begin{array}{c}
k \\
i
\end{array}\right)(k+i) \\
& =\sum_{i=0}^{k-1}(-1)^{i} 2^{2 k-2 i-1}\left(\begin{array}{c}
k \\
i
\end{array}\right)+\sum_{i=0}^{k-1}(-1)^{i} 2^{2 k-2 i-1}\left(\begin{array}{c}
k-1 \\
i-1
\end{array}\right) \\
& =\frac{1}{2}\left((4-1)^{k}-(-1)^{k}\right)-\frac{1}{2}\left((4-1)^{k-1}-(-1)^{k-1}\right) \\
& =3^{k-1}+(-1)^{k-1} .
\end{aligned}
$$

As for the second assertion of Theorem 4 , let $B_{k}=\left[b_{i j k}\right]$ be the $k \times k$ matrix with entries

$$
b_{i j k}=\left(\begin{array}{c}
j+k-i \\
i
\end{array}\right)+\left(\begin{array}{c}
j+k-i-1 \\
i-1
\end{array}\right)=\left(\begin{array}{c}
j+k-i \\
i
\end{array}\right) \frac{j+k}{j+k-i} \quad(0 \leq j, k<k) .
$$

Thus

$$
\operatorname{det} A_{k}=\operatorname{det} B_{k} \times \prod_{i=0}^{k-1}\left((-1)^{i} 2^{2 k-2 i-1}\right)=\varepsilon_{k} 2^{k^{2}} \operatorname{det} B_{k}
$$

so it suffices to show that $\operatorname{det} B_{k}=1$. We induct on $k$. We know that $B_{1}=[1]$, so $\operatorname{det} B_{1}=1$. Let $\mathbf{b}_{0 k}, \ldots, \mathbf{b}_{k-1 k}$ denote the columns of $B_{k}$. Our first task is to show that if $0<j<k$, then

$$
\mathbf{b}_{j k}-\mathbf{b}_{j-1 k}=\left[\frac{\mathbf{0}}{\mathbf{b}_{j-1 k-1}}\right] \text {. }
$$

To this end we note first that $b_{0 j k}=1$ for all $j$, so that $b_{0 j k}-b_{0 j-1 k}=0$ for $0<j<k$. If $2 i \leq j+k-1$, then

$$
\begin{aligned}
b_{i j k}-b_{i j-1 k}= & \left(\begin{array}{c}
j+k-i \\
i
\end{array}\right)+\left(\begin{array}{c}
j+k-i-1 \\
i-1
\end{array}\right) \\
& -\left(\begin{array}{c}
j+k-i-1 \\
i-1
\end{array}\right)-\left(\begin{array}{c}
j+k-i-2 \\
i-2
\end{array}\right) \\
= & b_{i-1 j-1 k-1} .
\end{aligned}
$$

If $2 i=j+k$, then $b_{i j-1, k}=0$, and so $b_{i j k}-b_{i j-1 k}=b_{i j k}=2=b_{i-1 j-1 k-1}$. If $2 i>j+k$, then $b_{i j k}=b_{i j-1 k}=b_{i-1 j-1 k-1}=0$. Thus we have $(6.1)$.

We now operate on $B_{k}$ as follows: We subtract column $\mathbf{b}_{k-2 k}$ from $\mathbf{b}_{k-1 k}$, then subtract $\mathbf{b}_{k-3 k}$ from $b_{k-2 k}$, and so on, until finally we subtract $\mathbf{b}_{0 k}$ from $\mathbf{b}_{1 k}$. The result is a matrix of the form

$$
\left[\begin{array}{cc}
1 & 0 \\
* & B_{k-1}
\end{array}\right]
$$

Thus $\operatorname{det} B_{k}=\operatorname{det} B_{k-1}$, so the induction is complete.

\section{Conjectures And Questions}

Based on some experimentation with the matrices $A_{k}$ and $B_{k}$, their characteristic polynomials and their eigenvalues, we propose the following:

Conjecture 1. All eigenvalues of $A_{k}$ are real.

Conjecture 2. If $k=2 r$, then $A_{k}$ has $r$ positive eigenvalues, and $r$ negative eigenvalues. If $k=2 r+1$, then $A_{k}$ has $r+1$ positive eigenvalues, and $r$ negative eigenvalues. 
Conjecture 3. If the negative eigenvalues of $A_{k}$ are replaced by their negatives, then the resulting numbers interlace with the positive eigenvalues, e.g., when $k=5$, the eigenvalues are $122.32,37.02$, $6.14,-18.59,-64.91$.

Conjecture 4. For each $k$, the characteristic polynomial $p_{k}$ is irreducible over $\mathbb{Q}$.

Conjecture 5. With $c_{k-r, k}$ defined in Theorem 1 and $\varepsilon_{r}$ defined in Theorem 4,

$$
\operatorname{sgn} c_{k-r, k}=\varepsilon_{r-1} \text {. }
$$

Conjecture 6. The zeros of $p_{k}$ interlace with those of $p_{k+1}$.

Conjecture 7. Let $B_{k}$ be defined as in the Proof of Theorem 4. The eigenvalues of $B_{k}$ are all positive real.

Conjecture 8 . The spectral radius of $A_{k}$ satisfies

$$
\rho\left(A_{k}\right)=\frac{3^{k}}{2}(1+o(1)) .
$$

Numerical computations suggest the stronger conjecture that $\rho\left(A_{k}\right)=\frac{1}{2} 3^{k}+O\left(k^{2}\right)$.

\section{REFERENCES}

[1] J.-P. Allouche and J. Shallit, The ubiquitous Prouhet-Thue-Morse sequence, in Sequences and their applications (Singapore, 1998), Springer Ser. Discrete Math. Theor. Comput. Sci., Springer, London, 1999, pp. 1-16.

[2] R. A. Askey, Orthogonal Polynomials and Special Functions, Regional conference series in applied mathematics 21, Society for Industrial and Applied Mathematics, Philadelphia, 1975.

[3] R. A. Askey, G. E. Andrews, R. Roy, Special Functions, Encyclopedia of Mathematics and its Applications 71, Cambridge University Press, Cambridge, 1999, pp. xvi+664.

[4] J.-P. Conze, A. Raugi, Fonctions harmoniques pour un opérateur de transition et applications Bull. Soc. Math. France, 118 (1990), 273-310.

[5] M. Drmota, C. Mauduit, J. Rivat, The sum-of-digits function of polynomial sequences, J. Lond. Math. Soc. (2) 84 (2011), no. 1, 81-102.

[6] E. Fouvry, C. Mauduit, Sommes des chiffres et nombres presque premiers, Math. Ann. 305 (1996), 571-599.

[7] C. F. Gauss, Disquisitiones generales circa seriem infinitam, Comm. Soc. Reg. Gött. 11, 1812; Werke, 3, $123-162$.

[8] A. O. Gelfond, Sur les nombres qui ont des propriétés additives et multiplicatives données, Acta Arith., 13 (1967/1968), pp. 259-265.

[9] H. W. Gould, Combinatorial Identities, a standardized set of tables listing 500 binomial coefficient summations, 1972, Morgantown WV, pp. viii+106.

[10] L. Hervé, Etude d'opérateurs quasi-compacts positifs. Applications aux opérateurs de transfert, Ann. Inst. H. Poincaré Probab. Statist. 30 (1994), no. 3, 437-466.

[11] L. Hervé, Construction et régularité des fonctions d'échelle, SIAM J. Math. Anal. 26 (1995), no. 5, 1361-1385.

[12] M. Keane, Generalized Morse sequences, Z. Wahrscheinlichkeitstheorie verw. Geb. 10 (1968), 259-265.

[13] K. Mahler, The Spectrum of an Array and Its Application to the Study of the Translation Properties of a Simple Class of Arithmetical Functions. II: On the Translation Properties of a Simple Class of Arithmetical Functions, J. of Math. Phys. Mass. Inst. Techn. 6 (1927), 158-163.

[14] C. Mauduit, Multiplicative properties of the Thue-Morse sequence, Period. Math. Hungar., 43 (2001), pp. 137153.

[15] C. Mauduit, J. Rivat, Sur un problème de Gelfond: la somme des chiffres des nombres premiers, Ann. Math. 171 (2010), 1591-1646.

[16] C. Mauduit and J. Rivat, La somme des chiffres des carrés, Acta Mathematica, 203 (2009), pp. 107-148.

[17] D. J. Newman, M. Slater, Binary digit distribution over naturally defined sequences Trans. Amer. Math. Soc. 213 (1975), 71-78.

[18] J. Riordan, Combinatorial identities, Wiley, New York, 1968.

[19] N. Wiener, The Spectrum of an Array and Its Applications to the Study of the Translation Properties of a Simple Class of Arithmetical Functions. I: The Spectrum of an Array, J. of Math. Phys. Mass. Inst. Techn. 6 (1927), 145-157. 
[20] M. A. Zaks, A. S. Pikowsky, J. Kurths On the correlation dimension of the spectral measure for the Thue-Morse sequence. Journ. Stat. Phys. (1997), 88, pp. 1387-1392.

[21] M. A. Zaks, A. S. Pikowsky, J. Kurths On the generalized dimensions for the Fourier spectrum of the ThueMorse sequence J. Phys. A: Math. Gen., 32, (1999) 1523-1530.

Université d'Aix-Marseille and Institut Universitaire de France, Institut de Mathématiques de Marseille, CNRS UMR 7373, 163 avenue de Luminy, Case 907, 13288 MARSEille Cedex 9, France E-mail address: mauduit@iml.univ-mrs.fr

Department of Mathematics, University of Michigan, 530 Church St., Ann Arbor, Mi 481091043, USA

E-mail address: hlm@umich.edu

Université D'Aix-Marseille, Institut de Mathématiques de Marseille, CNRS UMR 7373, 163 avenue de Luminy, Case 907, 13288 MarSeille Cedex 9, France

E-mail address: joel.rivat@univ-amu.fr 